\title{
Effect of a Low-Level Laser on Liposomal Doxorubicin Efficacy in a Melanoma Cell Line
}

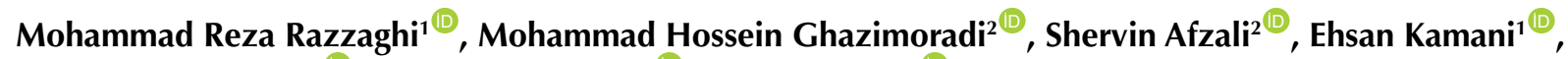 \\ Ezeddin Mohajerani $^{3^{\circledR}}$, Afshan Shirkavand ${ }^{\mathbb{D}^{\mathbb{D}}}$, Shirin Farivar ${ }^{2^{*} \mathbb{D}}$ \\ ${ }^{1}$ Laser Application in Medical Sciences Research Center, Shahid Beheshti University of Medical Sciences, Tehran, Iran \\ ${ }^{2}$ Department of Cell and Molecular Biology, Faculty of Life Sciences and Biotechnology, Shahid Beheshti University, \\ Tehran, Iran \\ ${ }^{3}$ POMP Lab, Photonics Department, Laser and Plasma Research Institute, Shahid Beheshti University, Tehran, Iran
}

\author{
*Correspondence to \\ Shirin Farivar, \\ Department of Cell and \\ Molecular Biology, Faculty of \\ Life Sciences and Biotechnology, \\ Shahid Beheshti University, \\ Tehran, Iran \\ Email: s_farivar@sbu.ac.ir
}

Received: February 16, 2021

Accepted: May 14, 2021

epublished: June 20, 2021

\begin{abstract}
Introduction: The cytotoxicity of chemotherapy drugs is a significant challenge in the way of surmounting cancer. Liposomal drug delivery has proven to be efficacious in increasing the function of the drugs. Its potential to accumulate drugs in the target site and enhance the efficiency of anticancer agents with lower doses hinders their cytotoxicity on normal healthy cells. Since the release of drugs from liposomes is not generally on a controlled basis, several studies have suggested that external stimuli including lasers could be used to induce controlled release and boost the efficiency of liposomal drug delivery systems (LDDSs).

Methods: The A375 cancer cell line was used and exposed to the liposomes containing doxorubicin in the presence of a low-level laser beam to investigate its effect on the liposomal stimuliresponsiveness release and its toxicity on cancer cells. So as to achieve that goal, Annexin V/PI was employed to analyze the number of cells that underwent apoptosis and necrosis.

Results: Here, we report the effect of laser irradiation on LDDSs. According to the results obtained from the annexin V/PI assay, the pattern of viability status has shifted, so that the number of preapoptotic cells treated with liposomal doxorubicin and a laser beam was more than that of cells treated with only liposomal doxorubicin.

Conclusion: The use of stimuli-responsive LDDSs, in this case, laser-responsive, has led to favorable circumstances in the treatment of cancer, offering enhanced cancer cell cytotoxicity.

Keywords: Low-level laser; Stimuli-sensitive liposomes; Cancer cytotoxicity; Liposomal drug delivery; Apoptosis.
\end{abstract}

\section{Introduction}

Most of the administered chemotherapy drugs are not distributed, particularly in an intended tissue, which leads to normal cell toxicity. ${ }^{1,2}$ Currently, liposomal drug delivery systems (LDDSs) are being exploited to hinder the complication mentioned above., ${ }^{3,4}$ Despite its usefulness in drug delivery compared to conventional drug administration, it still has some disadvantages, including random drug leakage at variable rates. ${ }^{5-7}$

Laser irradiation is being used as a newly emerging technique that makes the delivery of organic and inorganic molecules to cells, tissues, and organs in a purposive manner. ${ }^{8}$ One of the most significant applications of this technique is the delivery of anti-cancer agents to cancerous cells. ${ }^{9}$ This might, in turn, reduce the side effects and enhance the specificity of drugs and their toxicity on cancer cells. ${ }^{10,11}$ Another significant application of a laser is the rejuvenation of cells, tissues, and organs, especially skin. In recent years, the cells have been exposed to various compounds by this mechanism, such as vitamin C, growth factors, antibiotics, and chemotropic drugs. ${ }^{8,11-14}$ Moreover, it was shown that laser-sensitive liposomes could be functionalized by the laser beam to enhance the release of the cargo leading to cancer cell death. ${ }^{15,16}$

The mentioned data pointing to laser stimulation could eventually induce cell apoptosis. ${ }^{17}$ As laser photomodulation and irradiation on cells is one of the most widespread applications of a laser in medicine and it is being frequently used in LDDSs for cancer patients, investigating its effect on liposomal drug release is of great importance. ${ }^{18,19}$ In this study, we analyzed the levels of drug uptake and cell apoptosis to determine the essence and reliability of a laser in LDDSs.

\section{Materials and Methods Cell Culture}

The A375 cell line was procured from the Iranian Biological Resource Center (IBRC). These cells were 
maintained in high glucose DMEM (Gibco) supplemented with $10 \%$ FBS (Gibco) and $1 \%$ pen-strep (Gibco) at $37^{\circ} \mathrm{C}$ in humidified air containing $5 \% \mathrm{CO}_{2}$. The cells were passaged when they reached $80 \%$ confluency. ${ }^{20} 24$ hours prior to the treatment, the A375 cells were harvested with $0.025 \%$ trypsin and $0.52 \mathrm{mM}$ EDTA, after which the cell count was performed using Trypan blue staining (0.4\%). For each well, $0.07^{\star} 10(6)$ cells were plated in a 24 -well cell culture plate and allowed to adhere. ${ }^{21}$

\section{Treatment}

Three groups of cells were separated. Group 1 was the cells treated with $2.5 \mathrm{mg} / \mathrm{mL}$ of liposome encapsulated doxorubicin (DOXIL) for 12 hours and further exposed to a laser (gallium aluminum arsenide diode laser $(\lambda=655 \mathrm{~nm}, 200 \mathrm{~mW})$ at $\left.5 \mathrm{~J} / \mathrm{cm}^{2}\right)$ (combination treated group), group 2 was the cells only treated with the same concentration of DOXIL (LD), and group 3 consisted of the cells without any treatment. ${ }^{22,23}$

\section{Intracellular Uptake}

To investigate the intracellular uptake and accumulation of liposomal doxorubicin, the A375 cells were incubated with DOXIL for 12 hours. Then, the cells were harvested using trypsin and centrifugation at $1500 \mathrm{rpm}$. The cells were collected, washed twice with PBS and stained with nuclei stain, DAPI, for 20 minutes at $4^{\circ} \mathrm{C}$ in the dark. The uptake of DOX was analyzed by the BD FACSLyric flow cytometry device.

\section{Annexin V/PI}

The procedure was done according to the manufacturer's (BioLegend) protocol. The cells were trypsinized and centrifuged. The pellet was suspended in a fixing buffer and centrifuged. The cells were washed with cold cell staining buffer twice to decrease non-specific binding. Subsequently, they were resuspended in annexin $\mathrm{V}$ binding buffer and stained with FITC Annexin V and propidium iodide. The analysis was performed by the BD FACSLyric flow cytometry device. ${ }^{24,25}$

\section{Results}

It has been reported that doxorubicin has fluorescence properties that emit red light at a specific wavelength. ${ }^{26,27}$ Thus, the cells treated with doxorubicin were analyzed for doxorubicin uptake. The result exerted significant doxorubicin uptake comparing to the control group. The control group showed low fluorescence intensity for the cells stained with DAPI on the wavelength in which doxorubicin emitted light (Figure 1).

Furthermore, the cells were tested with Annexin V/ PI proliferation assay. The viability state of the cells was determined by dividing the graph into four quadrants, indicating whether the cells undergo apoptosis, necrosis, or are viable. In the combination treated group, the percentage of the cells located in the lower-left quadrant (Q4), evaluated as healthy cells, vs. apoptotic Population (Q3: early apoptotic and Q2: late apoptotic) is 76.3\%: $23.22 \%$. By comparison, healthy cells population vs. apoptotic population is $80.4 \%$ : $19.09 \%$, and $95.2 \%$ : $3.92 \%$ for LD and control groups respectively (Figure 2). The apoptotic population percentage difference of the combination treated group with LD and control groups is approximately $4 \%$ and $20 \%$, respectively. Indeed, this change reveals that the viability status of A375 cells slightly shifts toward apoptosis when the release of doxorubicin from liposomes is induced by a laser. That is to say, the apoptosis induction capability of doxorubicin enhances to an extent of $27 \%$ when it is coupled with laser emission.

To further clarify the variation made by each treatment in this observation, notable differences could be observed in the pre-apoptotic cell population of the combination treated group versus LD and control groups (a difference of $5.6 \%$ and $14.5 \%$ respectively). Despite the escalation of the number of pre-apoptotic cells, the percentage of late apoptotic cells in the LD group was negligibly higher than the combined group, with a $1.47 \%$ difference (Figure $3 b$, c).

\section{Discussion}

Currently, LDDSs are generally being used extensively as the primary option for the treatment of cancer. ${ }^{1}$ These approaches have proven to be successful by increasing therapeutic efficacy and hindering normal cell toxicity. ${ }^{28}$ However, LDDSs are not efficient altogether because of their incomplete drug release and random drug leakage. ${ }^{29}$

The role of lasers and light photobiomodulation has not fully been uncovered, and it remains enigmatic. However, they have proven to be somewhat efficacious when they are employed as external inducers that provide controlled release of drugs with higher efficiency in LDDSs. ${ }^{30}$ Yao et al have utilized a light-sensitive liposome to elucidate the effect of light-sensitive LDDSs to overcome doxorubicin resistance by enhancing the induction of apoptosis. Near-

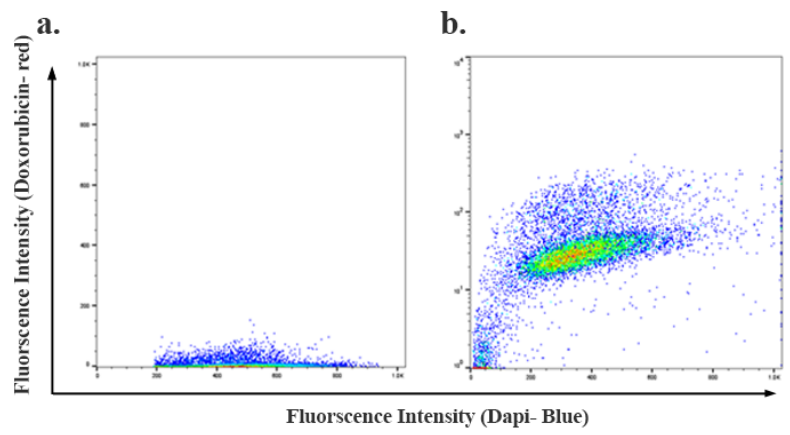

Figure 1. Flow Cytometry Analysis of Doxorubicin Uptake of the Cells Stained With Nuclei Stain, DAPI. (a) Control group (b) Doxorubicin-treated cells 

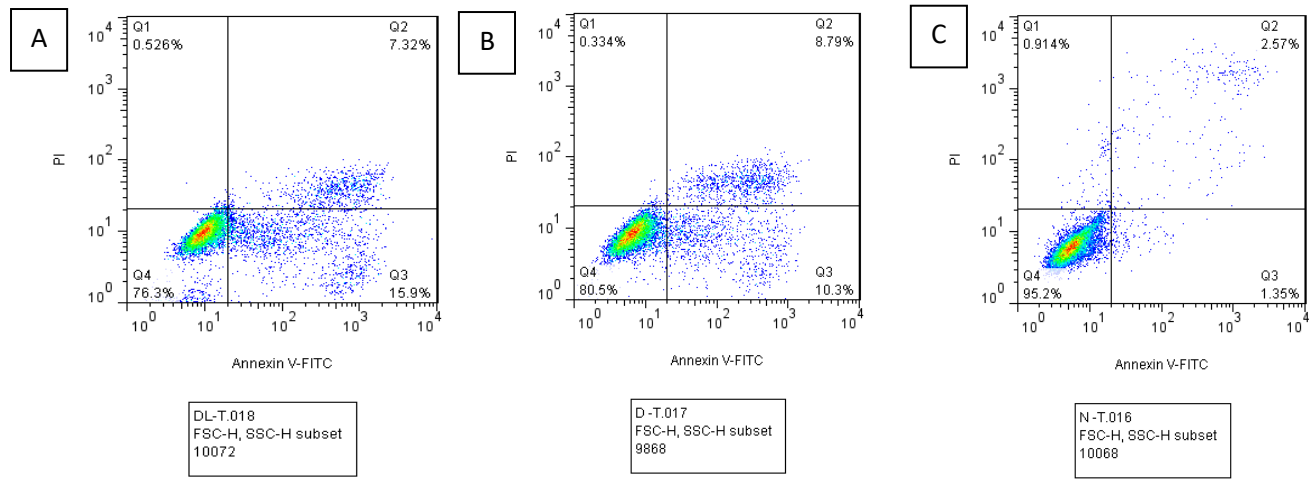

Figure 2. Annexin V/PI-Based Flow-Cytometric Assay: (A) combination treated group; (B) LD group; (C) control group.

a.

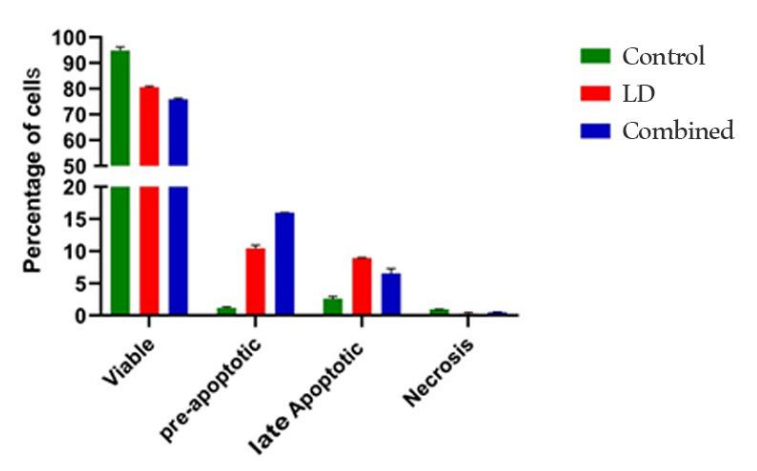

b.

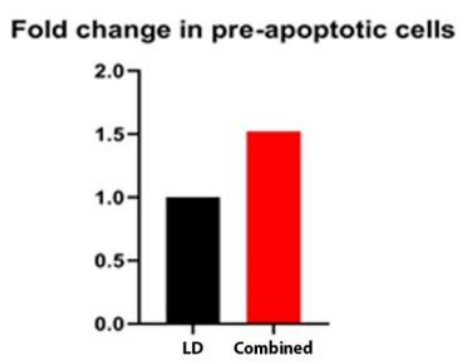

c.

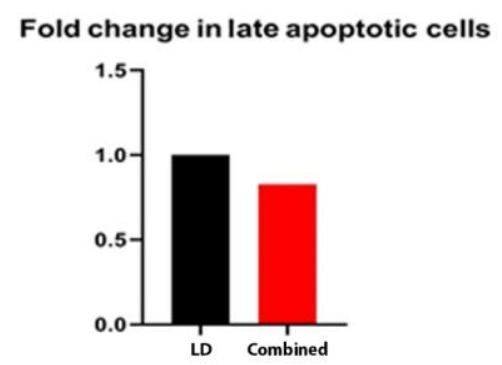

Figure 3. Visualized Changes in the Percentage of Four Quadrants of Annexin V/PI Flow Cytometry Assay. (a) Comparison of each quadrant in the three groups, and fold change in (b) pre-apoptotic cells and (c) late apoptotic cells of combined and LD groups.

infrared (NIR) light was used to control the induction of doxorubicin release from NIR-sensitive Azobenzeneliposomes. They have shown that 10-minute incubation with doxorubicin followed by NIR exposure enhances the rate of apoptosis compared to no NIR exposure and longer incubation time. ${ }^{6}$ Furthermore, Yavlovich et al have shown that $514 \mathrm{~nm}$ laser treatment has affected doxorubicin release from liposomes based on the wavelength of the beams and laser treatment increased cancer cells cytotoxicity to $49 \%, 51 \%$, and $64 \%$ when 80 , 160 and 200 nanogram liposomal doxorubicin was added to the samples. ${ }^{31}$ Similarly, we evaluated the early effect of a low-level laser on AT375 cells, a melanoma cell line. Our data and the performed assay analysis displayed an escalation in the occurrence rate of apoptosis when DOXIL treatment is accompanied by laser irradiation. To define our findings, it was shown that a slight increase in the pre-apoptotic population of cells occurred in the combination treated group in comparison with the LD group. However, a sharp increase in the number of pre-apoptotic cells is followed by a minor decrease in the percentage of cells in the late apoptotic state. This outcome may enhance the efficiency of LDDSs. Although the changes might not be significantly distinct, they 
can show different results in different kinds of cancers and could be exploited to reduce the cytotoxicity of the chemotherapeutic drugs.

\section{Conclusion}

Despite the advances toward better drug delivery systems, there are still some aspects that need to be considered in order to improve the LDDSs. Our data showed that lowpower laser administration could impede random drug leakage in LDDSs, and this could lead to an increased number of pre-apoptotic cells and cancer cell cytotoxicity. Further research is required for the optimization of laser exposure time and finding the relevant dosage of drug combinations which are more efficient and provide lower side effects. Moreover, it is of value to augment our understanding of LDDSs drug leakage.

\section{Ethical Considerations}

The study protocol was approved by the ethical committee of Shahid Beheshti University of Medical Sciences (IR.SBMU. RETECH.REC.1396.860).

\section{Conflict of Interests}

The authors declare there are no conflicts of interest regarding the publication of this article

\section{Acknowledgements}

The authors would like to thank the Laser Application in Medical Sciences Research Center for supporting this work.

\section{References}

1. Olusanya TOB, Haj Ahmad RR, Ibegbu DM, Smith JR, Elkordy AA. Liposomal Drug Delivery Systems and Anticancer Drugs. Molecules. 2018;23(4):907. doi:10.3390/ molecules23040907

2. Uddin MS, Ju J. Effect of crosslinking agents on drug distribution in chitosan hydrogel for targeted drug delivery to treat cancer. J Polym Res. 2020;27(3):1-10. doi:10.1007/ s10965-020-02059-8

3. Man F, Gawne PJ, R TMdR. Nuclear imaging of liposomal drug delivery systems: A critical review of radiolabelling methods and applications in nanomedicine. Adv Drug Deliv Rev. 2019;143:134-60. doi:10.1016/j.addr.2019.05.012

4. Lukowski JK, Weaver EM, Hummon AB. Analyzing liposomal drug delivery systems in three-dimensional cell culture models using MALDI imaging mass spectrometry. Anal Chem. 2017;89(16):8453-8. doi:10.1021/acs. analchem.7b02006

5. Gubernator J. Active methods of drug loading into liposomes: recent strategies for stable drug entrapment and increased in vivo activity. Expert Opin Drug Deliv . 2011;8(5):565-80. doi:10.1517/17425247.2011.566552

6. Yao C, Wang P, Li X, Hu X, Hou J, Wang L, et al. Nearinfrared-triggered azobenzene-liposome/upconversion nanoparticle hybrid vesicles for remotely controlled drug delivery to overcome cancer multidrug resistance. $A d v$ Mater. 2016;28(42):9341-8. doi:10.1002/adma.201503799

7. Sopyan I, Gozali D. A review: a novel of efforts to enhance liposome stability as drug delivery approach.
Systematic Reviews in Pharmacy. 2020;11(6). doi:10.31838/ srp.2020.6.85

8. Mester E, Mester AF, Mester A. The biomedical effects of laser application. Lasers Surg Med. 1985;5(1):31-9. doi:10.1002/lsm.1900050105

9. Wenande E, Olesen UH, Boesen MR, Persson DP, Lerche $\mathrm{CM}$, Sturup S, et al. Laser-assisted delivery enhances topical uptake of the anticancer agent cisplatin. Drug Deliv. 2018;25(1):1877-85. doi:10.1080/10717544.2018.1534896

10. Goldman L. Laser Cancer Research. Springer Science \& Business Media; 2012.

11. Rhodes K, Clark I, Zatcoff M, Eustaquio T, Hoyte KL, Koller MR. Cellular laserfection. Methods Cell Biol. 2007;82:30933. doi:10.1016/S0091-679X(06)82010-8

12. Karu TI, Pyatibrat LV, Kalendo GS, Esenaliev RO. Effects of monochromatic low-intensity light and laser irradiation on adhesion of HeLa cells in vitro. Lasers Surg Med. 1996;18(2):171-7. doi:10.1002/(SICI)10969101(1996)18:2<171::AID-LSM7>3.0.CO;2-P

13. Murphy-Chutorian D, Mueller RL, Harman SD, Daniel SA, Witham L, Richardson B, inventors; Eclipse Surgical Technologies Inc, assignee. Laser delivery means adapted for drug delivery. United States patent US 5,999,678. 1999 Dec 7.

14. Farivar S, Malekshahabi T, Shiari R. Biological effects of low level laser therapy. J Lasers Med Sci. 2014;5(2):58.

15. Khosroshahi M, Ghazanfari L, Hasannejad Z. Effect of laser wavelengths on drug release with and without gold nanoshells and magnetic guidance on uptake by cancer cells. J Nanomed Res. 2017;6. doi:10.15406/jnmr.2017.06.00152

16. Guo Y, Chen Y, Han P, Liu Y, Li W, Zhu F, et al. Biocompatible chitosan-carbon nanocage hybrids for sustained drug release and highly efficient laser and microwave coirradiation induced cancer therapy. Acta Biomater. 2020;103:237-46. doi:10.1016/j.actbio.2019.12.010

17. Yu S, Huang G, Yuan R, Chen T. A uPAR targeted nanoplatform with an NIR laser-responsive drug release property for tri-modal imaging and synergistic photothermal-chemotherapy of triple-negative breast cancer. Biomater Sci. 2020;8(2):720-38. doi:10.1039/ c9bm01495k

18. Goldman L. The Biomedical Laser: Technology and Clinical Applications. Springer Science \& Business Media; 2013.

19. Raulin C, Karsai S. Laser and IPL Technology in Dermatology and Aesthetic Medicine. Springer Science \& Business Media; 2011.

20. Balavandi Z, Neshasteh-Riz A, Koosha F, Eynali S, Hoormand $M$, Shahidi $M$. The use of $ß$-Elemene to enhance radio sensitization of A375 human melanoma cells. Cell J (Yakhteh). 2020;21(4):419. doi:10.22074/cellj.2020.6326

21. Zhang HL, Si LB, Zeng A, Long F, Qi Z, Zhao R, et al. MicroRNA-21 antisense oligonucleotide improves the sensitivity of A375 human melanoma cell to cisplatin: an in vitro study. J Cell Biochem. 2018;119(4):3129-41. doi: $10.1002 /$ jcb. 26455

22. de Lima RdN, Vieira SS, Antonio EL, de Carvalho PdTC, de Paula Vieira R, Mansano BSDM, et al. Low-level laser therapy alleviates the deleterious effect of doxorubicin on rat adipose tissue-derived mesenchymal stem cells. $J$ 
Photochem Photobiol B. 2019;196:111512. doi:10.1016/j. jphotobiol.2019.111512

23. Tang Y, McGoron AJ. Combined effects of laser-ICG photothermotherapy and doxorubicin chemotherapy on ovarian cancer cells. J Photochem Photobiol B. 2009;97(3):138-44. doi:10.1016/j.jphotobiol.2009.09.001

24. Tanaka J, Hirano K, Sakamoto Y, Sugahara-Tobinai A, Endo $\mathrm{S}$, Ito-Matsuoka $\mathrm{Y}$, et al. Intravenous immunoglobulin suppresses IL-10 production by activated B cells in vitro. Open J Immunol. 2012;2(4): 149-160. doi:10.4236/ oji.2012.24019

25. Kinsella S, Evandy CA, Cooper K, Iovino L, Paul Cd, Hopwo KS, et al. Attenuation of homeostatic signaling from apoptotic thymocytes triggers a global regenerative response in the thymus. bioRxiv. 2020. doi:10.1101/2020.08.31.275834

26. Kauffman MK, Kauffman ME, Zhu H, Jia Z, Li YR. Fluorescence-Based Assays for Measuring Doxorubicin in Biological Systems. React Oxyg Species (Apex). 2016;2(6):432-9. doi:10.20455/ros.2016.873

27. Hu J, Zhan S, Wu X, Hu S, Wu S, Liu Y. Core/shell upconversion nanoparticles with intense fluorescence for detecting doxorubicin in vivo. RSC advances. 2018;8(38):21505-12. doi:10.1039/C8RA02928H

28. Allen TM, Martin FJ. Advantages of liposomal delivery systems for anthracyclines. Semin Oncol. 2004;31(suppl.13):5-15. doi:10.1053/j. seminoncol.2004.08.001

29. Fuse T, Tagami T, Tane M, Ozeki T. Effective lighttriggered contents release from helper lipid-incorporated liposomes co-encapsulating gemcitabine and a watersoluble photosensitizer. Int J Pharm. 2018;540(1-2):50-6. doi:10.1016/j.ijpharm.2018.01.040

30. Wu G, Mikhailovsky A, Khant HA, Fu C, Chiu W, Zasadzinski JA. Remotely triggered liposome release by near-infrared light absorption via hollow gold nanoshells. J Am Chem Soc. 2008;130(26):8175-7. doi:10.1021/ ja802656d

31. Yavlovich A, Singh A, Blumenthal R, Puri A. A novel class of photo-triggerable liposomes containing DPPC: DC8, 9PC as vehicles for delivery of doxorubcin to cells. Biochim Biophys Acta. 2011;1808(1):117-26. doi:10.1016/j. bbamem.2010.07.030 